\title{
Single-Cell WGA-Next-Generation Sequencing and its Applications in Microbial Ecology
}

\section{Sat Pal Singh Bisht ${ }^{\star}$}

Department of Zoology, Kumaun University, Nainital, Uttarakhand, India

Molecular microbial ecology begins in the year 1990 with the direct amplification and sequencing of $16 \mathrm{~S}$ rRNA genes from the environment. This technology revolutionized microbial ecology and changed the way we study prokaryotes in the environment. The adoption of molecular tools by microbial ecologists has rapidly enhanced our knowledge of prokaryote abundance, diversity and function. The ultimate goal of microbial ecology research is to elucidate the factors that control the extreme heterogeneity of microbial population in the environment by investigating the interaction of microorganisms with each other and the biotic and abiotic characteristics of the environment. The development of next generation sequencing technology and decreasing cost of sequencing in the last decade made possible to study the dynamics of microbial communities as well as dynamics of thousands of different microbial populations simultaneously.

The complexity of microbial communities varies in each and every ecosystem of this planet. The vast majority of microbial species remain uncultivated till date; about half of all known bacterial phyla were identified only from their $16 \mathrm{~S}$ ribosomal RNA gene sequences. With the dawn of single-cell genome sequencing it is possible to access the genomes of uncultivated organisms, determining the basis of microbial community functions and fundamental aspects of microbial population biology. Single-cell sequencing provides information on low-abundance species of an environment that are difficult to culture. Single-cell sequencing depends on the physical/ manual isolation of individual cells and the use of these cells as templates for PCR amplification and sequencing of specific genomic loci. Flow cytometry and Fluorescence-Activated Cell Sorting (FACS) have become popular platforms for single-cell genomic analysis. Micromanipulation approaches differ from conventional cell isolation approaches in that targeted cells are identified first and then isolated. Single-cell whole genome-sequencing work flows require the most demanding sample preparation: cell isolation, cell lysis, amplification, WGA product screening and sequencing. Amplification and sequencing of genomic DNA from targeted species allowed the physiology and ecology of cells to be inferred. Single-cell sequencing promises to provide direct access to fine-scale heterogeneity in complex microbial populations by resolving and linking 'fuzzy' diversity across whole genomes sequenced from individual cells. Several biological phenomena can be accessible for investigation with single-cell sequencing i.e. genetics and metabolism of as-of-yet unculturable microbes, the clonal diversity within cancer and tumour evolution, the role of genetic mosaicism in the biology of multicellular organisms, the genomic variation in gamete cells and embryos.

Single cell genomics allows to analyze the metabolic potential and evolutionary histories of the so called microbial dark matter- those 99\% of microbial types that constitute the majority of biomass and biodiversity on our planet but have not been amenable to studies using traditional microbiology methods. Analysis of single-cell genomes often provides information of pathways of potential interest in novel drug discovery such as polyketide or antibiotic synthesis. Single cell sequencing was selected as "method of the year for 2013" by nature methods for its potential in understanding life one cell at a time.
*Corresponding author: Sat Pal Singh Bisht, Department of Zoology, Kumaun University, Nainital, Uttarakhand, India, Tel: 91-5942-23678; E-mail: sps.bisht@gmail.com Received September 29, 2015; Accepted October 05, 2015; Published October 07, 2015

Citation: Bisht SPS (2015) Single-Cell WGA-Next-Generation Sequencing and its Applications in Microbial Ecology. Next Generat Sequenc \& Applic 2: 120. doi:10.4172/2469-9853.1000120

Copyright: (c) 2015 Bisht SPS. This is an open-access article distributed under the terms of the Creative Commons Attribution License, which permits unrestricted use, distribution, and reproduction in any medium, provided the original author and source are credited. 